\title{
Local Lattice Match for Commensurate State of Graphene/h-BN van der Waals Heterostructure with TEM Analysis
}

$\mathrm{Na}$ Yeon $\mathrm{Kim}^{1,2}$, Jung Hwa Kim${ }^{1}$ and Zonghoon Lee ${ }^{1,2}$

1. School of Materials Science and Engineering, Ulsan National Institute of Science and Technology (UNIST), Ulsan Metropolitan City 44919, Republic of Korea

2. Center for Multidimensional Carbon Materials, Institute for Basic Science (IBS), Ulsan Metropolitan City 44919, Republic of Korea

The transition to commensurate state of van der Waals heterostructures of 2D materials overcomes the inherent drawbacks on pure 2D crystals and evokes peculiar properties with spontaneous alternation of the periodicity in each 2D layer and 2D van der Waals superstructures [1]. However, the lattice mismatch between graphene and h-BN is extremely small around $0.05 \AA$ and the commensurate transition occurs only around the AB-stacked area where carbon atoms are located above the boron (B) atoms when graphene and h-BN are aligned along almost identical orientations [2,3]. Therefore, local and uneven transitions to the commensurate state are difficult to distinguish.

In the present study, we propose the evidence of a local transition to the commensurate state of graphene /h-BN van der Waals heterostructure $(\mathrm{G} / \mathrm{h}-\mathrm{BN})$ through moiré superstructure analysis using dark field transmission electron microscopy [4]. The moiré fringes are locally widened and zigzagged only when twist angle is close to 0 as presented in Figure 1, which is caused by local twisting and stretching in the $\mathrm{AB}$-stacked configurations of the graphene lattices, in other words, enlargement of the perfect ABstacked area marked with red in Figure 2. Furthermore, we statistically characterized the reduced lattice mismatch as a function of a decreased twist angle. Therefore, this moiré superstructure analysis with dark field transmission electron microscopy can reveal the local structural variation of G/h-BN even under a low magnification and provide experimental evidence of the theoretically predicted relation with respect to the stacking configurations for discerning the altered electronic properties of $\mathrm{G} / \mathrm{h}-\mathrm{BN}$ only with a low twist angle.

\section{References:}

[1] B. Hunt et al, Science 340 (2013), p.1427-1430.

[2] C. R. Woods et al, Nature Physics 10 (2014) p.451-456.

[3] M. Yankowitz et al, Nature Communications 7 (2016), p.13168.

[4] N. Y. Kim et al, ACS Nano 11 (2017), p. 7084-7090.

[5] The authors acknowledge funding from the Institute for Basic Science (IBS-R019-D1), a National Research Foundation of Korea (NRF) grant funded by the Korean government (MSIP) (No.2015R1A2A2A01006992), and the Nano Material Technology Development Program (2012M3A7B4049807). 

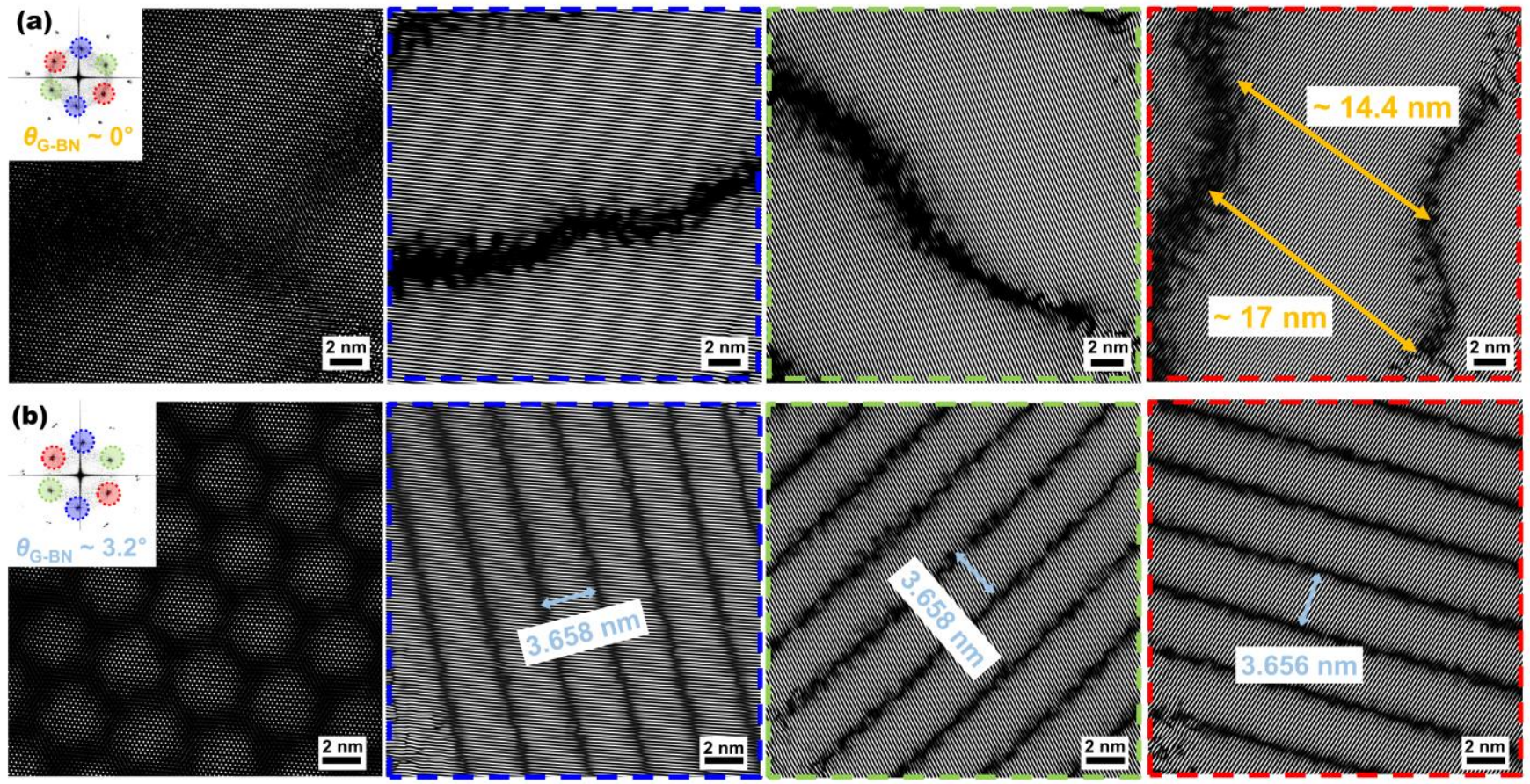

Figure 1. Widening and bending of the moire fringes of $\mathrm{G} / \mathrm{h}-\mathrm{BN}$ only at low twist angle $\left(\theta_{\mathrm{G}-\mathrm{BN}}\right)$. ARTEM images of $\mathrm{G} / \mathrm{h}-\mathrm{BN}$ and the corresponding selected lattice fringe images, formed by masking diffraction reflections on the FFTs with (a) $\theta_{\mathrm{G}-\mathrm{BN}} \approx 0^{\circ}$ and (b) $\theta_{\mathrm{G}-\mathrm{BN}}=3.2^{\circ}$. The bright and dark moiré fringes are caused by constructive and destructive interferences, respectively, between the graphene and h-BN lattice fringes [4].
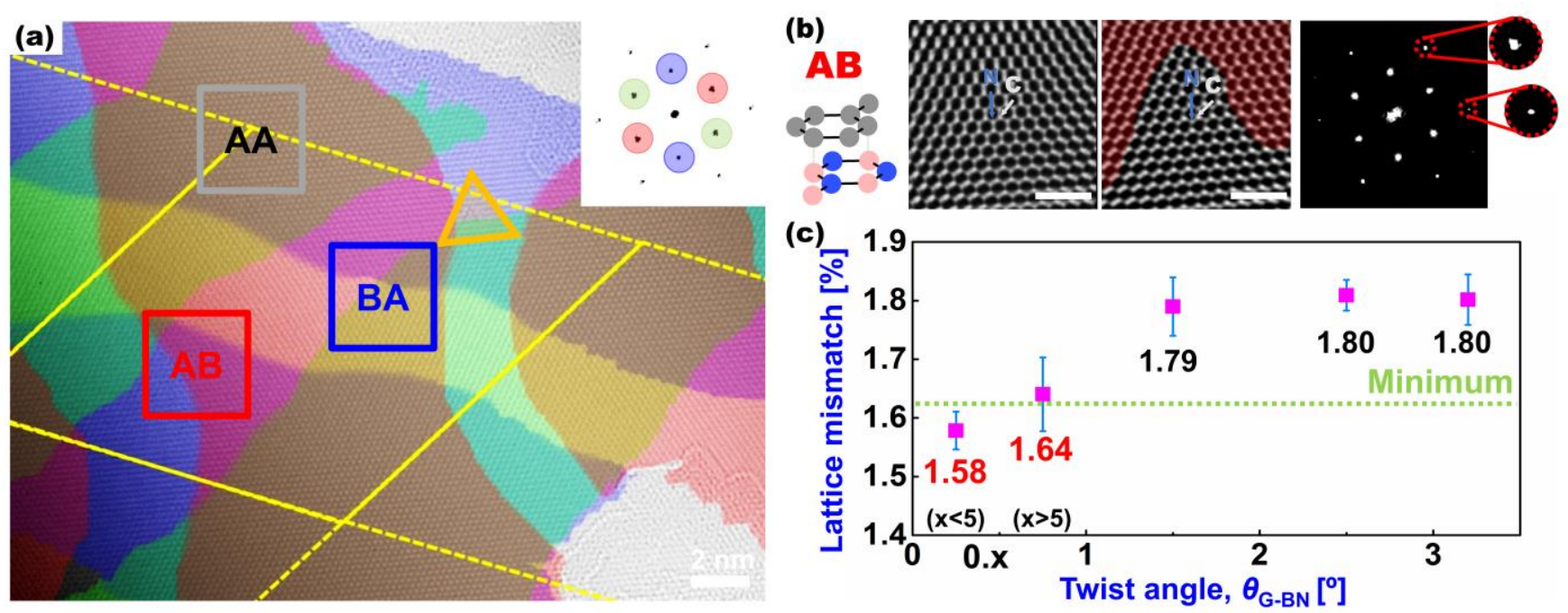

Figure 2. Transition to commensurate state in the AB-stacked area of G/h-BN. (a) Colored moiré fringes superimposed on an AR-TEM image with widening and zigzagged morphology depending on the stacking configurations when $\theta_{\mathrm{G}-\mathrm{BN}}=0.4^{\circ}$. (b) Evidence of the enlarged AB-stacked area by comparing simulated AR-TEM image and experimental one with corresponding FFT (c) Average lattice mismatch between graphene and $\mathrm{h}-\mathrm{BN}$ versus twist angle (the trend decreases below $\theta_{\mathrm{G}-\mathrm{BN}}=1^{\circ}$ ) [4]. 